\title{
Los Estudios de Comunicación y Creación Audiovisual en la Universidad española: el caso de Castilla y León_
}

\author{
The Studies of Communication and Audiovisual Creation in Spanish \\ Universities: The Case of Castilla y León
}

Beatriz González de Garay Domínguez

y María Marcos Ramos

Los Estudios de Comunicación Audiovisual en la Universidad española

La importancia de los estudios de Comunicación Audiovisual en España ha ido aumentando a medida que el sector audiovisual demostraba su importancia en la sociedad española. Cada vez son más las universidades que apuestan por ofrecer estudios relacionados con el audiovisual, bien en el Grado de Comunicación Audiovisual o bien en grados afines. Este artículo tiene como objetivo fundamental analizar qué se estudia en Comunicación Audiovisual, por lo que se hará un análisis de los planes de estudios y un repaso de las principales universidades que imparten este grado, las notas de acceso y el número de alumnos admitidos. También serán objeto de estudio la legislación por la que se rige el Grado de Comunicación Audiovisual y las competencias que desarrollan los estudiantes de cara a incorporarse al mundo profesional. Se hará especial hincapié en la implantación de este grado en la Comunidad Autónoma de Castilla y León.

[...] España es uno de los primeros países de Europa en incorporar los estudios oficiales de cinematografía a la universidad, este proceso se realizará con mucha lentitud desde los años sesenta hasta los ochenta, en los que se despliega un amplio y disperso abanico de estudios bajo denominaciones muy diversas y, en ocasiones, poco clarificadoras de las materias a impartir por el profesorado y a aprender por el estudiante. Sin embargo, la gran demanda social de enseñanzas sobre cine y televisión que se manifiesta durante los años ochenta va a estructurarse en el sistema universitario español con la apertura de Facultades de Ciencias de la Información y de la Comunicación en diversas universidades del Estado, que constituyen secciones especifficas de Imagen, con licenciaturas en Comunicación Audiovisual de ciclo corto (tres años) o largo (cinco años). (Rodríguez Merchán, 2007: 17)

Los estudios superiores sobre audiovisuales en España tienen su origen en la Escuela Oficial de Cinematografía (Rodríguez Merchán, 2007). Más adelante y “al amparo de la Ley General de Educación de 1970", José Luis Villar Palasí, entonces Ministro de Educación, emite un decreto (Real Decreto de 13/08/1971) mediante el cual se modifican los estudios "referidos al periodismo y demás medios de comunicación social", incorporándolos a la "nueva estructura universitaria y la dinámica general de los procesos educativos". Quizá por razones más políticas que académicas, tal 


\section{taphiya $\mathbf{4 8}$}

como se ha intentado explicar antes, el citado decreto sienta las bases para la creación de las Facultades de Ciencias de la Información como únicos centros capacitados legalmente para impartir "las enseñanzas correspondientes a periodismo, cinematografía, televisión, radiodifusión y publicidad" (Rodríguez Merchán, 2007, pp. 16-17).

El título de Licenciado en Comunicación Audiovisual fue regulado por el Real Decreto 1427/1991, de 30 de agosto, en el que se establecieron las directrices generales y el plan de estudios conducente a la obtención del mismo'. Las enseñanzas para la obtención del título oficial de Licenciado en Comunicación Audiovisual implicaban formación en el campo de la elaboración informativa y de la creación, producción y realización en los diversos medios de comunicación audiovisual y, para ello, se instaba a las universidades que lo implantasen a articular los estudios en enseñanzas de primer y segundo ciclo, con una duración total de entre cuatro y cinco años, y una duración por ciclo de, al menos, dos años.

Los distintos planes de estudios conducentes a la obtención de título oficial de Licenciado en Comunicación Audiovisual determinarían, en créditos, la carga lectiva global, que en ningún caso sería inferior a 300 créditos, ni superior al máximo de créditos que para los estudios de primer y segundo ciclo permitía el Real Decreto 1497/1987, por el que se establecen directrices generales comunes a los planes de estudio de los títulos universitarios de carácter oficial y validez en todo el territorio nacional. En ningún caso, el mínimo de créditos de cada ciclo sería inferior a 120. Por otro lado, la carga lectiva establecida en el plan de estudios oscilaría entre veinte y treinta horas semanales, incluidas las enseñanzas prácticas. Bajo ningún concepto, la carga lectiva de la enseñanza teórica podría superar las quince horas semanales. En cuanto a los créditos, estos estaban definidos por el artículo 2.7 del Real Decreto 1497/1987 como la unidad de valoración de las enseñanzas universitarias y correspondían a 10 horas de clase teórica y/o práctica.

Se estableció, así mismo, la relación de las materias troncales: separadas en dos ciclos y con la carga docente dividida en créditos teóricos y prácticos. Además, por cada materia troncal se establecieron diferentes áreas de conocimiento, tal y como se puede ver en la Tabla 1.

\begin{tabular}{llll}
\hline $\begin{array}{l}\text { Relación de materias troncales (por orden } \\
\text { alfabético) }\end{array}$ & Créditos & Áreas de conocimiento \\
\cline { 2 - 3 } & Teóricos Prácticos Total &
\end{tabular}

Primer ciclo

Análisis del entorno social y de su evolución histórica: profundización en las realidades del mundo contemporáneo en sus dimensiones política, económica y social. Evolución histórica.
7

1

8 Ciencia Política y de la Administración, Historia Contemporánea, Historia del Derecho y de las Instituciones, Historia del Pensamiento y de los Movimientos Sociales y Políticos, Historia e Instituciones Económicas y Sociología.

6

Comunicación e información audiovisual: estudio y capacitación en la tecnología, el análisis y la expresión de formas y medios audiovisuales.

\footnotetext{
${ }^{1}$ Real Decreto 1427/1991, de 30 de agosto, por el que se establece el título universitario oficial de Licenciado en Comunicación Audiovisual y las directrices propias de los planes de estudios conducentes a la obtención de aquel. BOE, núm. 243, de 10 de octubre de 1991, páginas 32901 a 32902.
} 


\begin{tabular}{|c|c|c|c|c|}
\hline $\begin{array}{l}\text { Comunicación e información escrita: } \\
\text { estudio y capacitación en la tecnología, el } \\
\text { análisis y la expresión en formas y medios } \\
\text { impresos y electrónicos. }\end{array}$ & 6 & 6 & 12 & $\begin{array}{l}\text { Comunicación Audiovisual y Publicidad } \\
\text { y Periodismo. }\end{array}$ \\
\hline $\begin{array}{l}\text { Documentación informativa: estudio y } \\
\text { análisis de los sistemas de documentación } \\
\text { utilizados en los medios de comunicación. }\end{array}$ & 3 & 3 & 6 & $\begin{array}{l}\text { Biblioteconomía y Documentación, } \\
\text { Comunicación Audiovisual y Publicidad } \\
\text { y Periodismo. }\end{array}$ \\
\hline $\begin{array}{l}\text { Lengua: introducción teórico-práctica al } \\
\text { conocimiento y uso de la lengua española o } \\
\text { de la otra lengua oficial propia de la } \\
\text { Comunidad Autónoma donde radique la } \\
\text { Universidad, a elección del alumno. }\end{array}$ & 4 & 4 & 8 & Filología correspondiente. \\
\hline $\begin{array}{l}\text { Publicidad y relaciones públicas: } \\
\text { introducción teórica y práctica a la } \\
\text { publicidad y las relaciones públicas y a sus } \\
\text { estructuras organizativas. }\end{array}$ & 6 & 6 & 12 & $\begin{array}{l}\text { Comunicación Audiovisual y } \\
\text { Publicidad. }\end{array}$ \\
\hline $\begin{array}{l}\text { Teoría de la comunicación y teoría de la } \\
\text { información: estudio de los elementos, } \\
\text { formas, procesos y estructuras de la } \\
\text { comunicación, así como de los métodos de } \\
\text { investigación en comunicación. Evolución } \\
\text { histórica. }\end{array}$ & 8 & 2 & 10 & $\begin{array}{l}\text { Comunicación Audiovisual y } \\
\text { Publicidad, Periodismo, Psicología } \\
\text { Social y Sociología. }\end{array}$ \\
\hline
\end{tabular}

\section{Segundo ciclo}

Derecho de la información: información y 5 2

7 Derecho Administrativo y Derecho derechos fundamentales. Plasmación en la Constitucional. Constitución Española. Régimen jurídico de la información y la comunicación. Estudio teórico y supuestos prácticos en mensajes, medios y sujetos.
Estructura del sistema audiovisual: descripción e investigación de la naturaleza e interrelaciones entre los sujetos de la comunicación audiovisual: autores, instituciones, empresas, medios, soportes y receptores. 4

$\begin{aligned} & \text { Narrativa audiovisual: sistemas y procesos } \\ & \text { de construcción y análisis de los relatos }\end{aligned}$
audiovisuales.




\begin{tabular}{|c|c|c|c|c|}
\hline $\begin{array}{l}\text { Producción y realización audiovisuales: } \\
\text { técnicas y procesos de creación y difusión } \\
\text { audiovisuales en sus diversas fases. }\end{array}$ & 4 & 9 & 13 & $\begin{array}{l}\text { Comunicación Audiovisual y } \\
\text { Publicidad. }\end{array}$ \\
\hline $\begin{array}{l}\text { Teoría de la comunicación audiovisual: } \\
\text { conceptuación y análisis de las } \\
\text { representaciones icónicas y acústicas y de } \\
\text { su evolución histórica. }\end{array}$ & 5 & 2 & 7 & $\begin{array}{l}\text { Comunicación Audiovisual y Publicidad } \\
\text { y Periodismo. }\end{array}$ \\
\hline
\end{tabular}

Tabla 1. Relación de materias troncales Título de Licenciado en Comunicación audiovisual (Real Decreto 1427/1991).

La Conferencia de Decanos de Facultades de Ciencias de la Comunicación, presidida por el profesor Marcial Murciano en calidad de Decano de la Facultad de Ciencias de la Comunicación de la Universidad Autónoma de Barcelona-, fue responsable de la realización del Libro Blanco de los títulos de Grado en Ciencias de la Comunicación, con el apoyo de la ANECA (2005). Su objetivo era analizar los estudios de comunicación que se desarrollaban en la universidad española (Periodismo, Comunicación Audiovisual y Publicidad y Relaciones Públicas) para crear una propuesta que permitiera adaptarlos a los parámetros que establece el marco europeo de convergencia en educación superior. En la Conferencia de Decanos, participaron cuarenta universidades, es decir, todas aquellas instituciones -públicas o privadas- que impartían al menos un título vinculado con las Ciencias de la Comunicación en aquel momento. El documento final que dio lugar al citado Libro Blanco fue aprobado por consenso unánime de los participantes en la reunión plenaria celebrada en Madrid, en la Universidad San Pablo CEU, el día 28 de octubre de 2004.

El documento se elaboró a partir de diferentes fuentes. En primer lugar, se realizó un censo de los datos básicos, como el año de inicio, la titularidad o el número de alumnos/as matriculados/as y licenciados/as de cada una de las universidades que ofrecían estudios de Comunicación, diferenciando cada una de las titulaciones. En segundo lugar, se llevó a cabo un análisis pormenorizado de los estudios de Periodismo, Comunicación Audiovisual y Publicidad y Relaciones Públicas en Europa -Alemania, Eslovaquia, Finlandia, Francia, Italia, Lituania, Portugal, Reino Unido, República Checa y Suecia- y se atendió a la evolución histórica de los estudios de Comunicación en España. En tercer lugar, se apoyó en un estudio basado en encuestas realizadas a cuatro colectivos: alumnado, profesorado, gestores universitarios y profesionales. Las diversas tareas fueron desempeñadas por las subcomisiones de Periodismo², Comunicación Audiovisual $\left.\right|^{3}$ y la de Publicidad y Relaciones Públicas.

La elaboración del Libro Blanco permitió concretar los perfiles profesionales básicos para cada titulación, los conocimientos disciplinares (saber), las competencias profesionales (saber hacer), las competencias académicas y

\footnotetext{
${ }^{2}$ Formada por el Dr. Josep Lluís Gómez Mompart, Universidad de Valencia; el Dr. Jaume Guillamet, Universidad Pompeu Fabra; el Dr. Javier Galán, Universidad Carlos III de Madrid; la Dra. Consolación Isart, San Pablo CEU; el Dr. Manuel Roglán, Universidad Antonio de Nebrija; y la Dra. María Jesús Casals, Universidad Complutense de Madrid.

3 Integrada por el Dr. Domènec Font, Universidad Pompeu Fabra; la Dra. Inmaculada Chacón, Universidad Europea de Madrid; el Dr. Juan Antonio García Galindo, Universidad de Málaga; el Dr. Pablo del Río, Universidad de Salamanca; el Dr. Emili Prado, Universidad Autónoma de Barcelona; el Dr. Enrique Bustamante, Universidad Complutense de Madrid; el Dr. Xaime Fandiño, Universidad de Vigo; y el Dr. Santos Zunzunegui, Universidad del País Vasco.

${ }^{4}$ Constituida por el Dr. Rafael López Lita, Universidad Jaume I; la Dra. Sara Magallón, Universidad de Barcelona; el Dr. Alfonso Sánchez-Tabernero, Universidad de Navarra; el Dr. Ubaldo Cuesta, Universidad Complutense de Madrid; el Dr. José María Ricarte, Universidad Autónoma de Barcelona; y el Dr. José Luis León, Universidad del País Vasco.
} 


\section{taphiya $\mathbf{4 8}$}

transversales (saber estar o ser), siguiendo las directrices marcadas por el Informe Delors (1996), en el que se indicaba que las demandas sociales requieren de una formación que desarrolle competencias básicas y específicas en los estudiantes y que esté vinculada a cuatro pilares de la educación: aprender a conocer, aprender a hacer, aprender a vivir juntos / aprender a vivir con los demás y aprender a ser.

En la Tabla 2 se puede ver la distribución de créditos en materias obligatorias del Grado en Comunicación y Creación Audiovisual de la Universidad de Salamanca, a modo de ejemplo, y los contenidos formativos mínimos del Libro Blanco con los que se corresponden. Las asignaturas obligatorias comprenden un total de 132 créditos ECTS, distribuidos en nueve materias y veintidós asignaturas. Cada asignatura obligatoria tiene 6 créditos. Se puede observar un especial peso de las Técnicas y procesos creativos de producción, realización y postproducción y las Tecnologías de los medios audiovisuales y multimedia.

\begin{tabular}{|c|c|c|}
\hline Créditos & $\begin{array}{l}\text { Nombre de la materia } \\
\text { obligatoria }\end{array}$ & $\begin{array}{l}\text { Contenidos formativos mínimos / Asignaturas obligatorias del Plan de estudios del } \\
\text { Grado CAAV }\end{array}$ \\
\hline 12 & $\begin{array}{l}\text { Teoría e historia de los medios } \\
\text { audiovisuales y nuevos soportes } \\
\text { multimedia (Contenido } \\
\text { obligatorio común) }\end{array}$ & $\begin{array}{l}\text { - Historia de los medios audiovisuales: fotografía, cine, radio, televisión, vídeo, } \\
\text { cómic. } \\
\text { - Fundamentos téenicos y expresivos en los lenguajes multimedia. } \\
\text { - Conocimiento histórico de los diferentes paradigmas relacionados con las teorías } \\
\text { de la comunicación audiovisual. } \\
\text { - Historia y estética de las representaciones visuales. } \\
\text { - Géneros audiovisuales y cultura de masas. } \\
\text { - Las empresas creadoras de producciones culturales y comunicativas y los diferentes } \\
\text { tipos de portales en red. } \\
\text { Asignaturas: Géneros audiovisuales (6ECTS) e Historia de los medios audiovisuales } \\
\text { (6ECTS). }\end{array}$ \\
\hline 6 & $\begin{array}{l}\text { Estructura y políticas de la } \\
\text { comunicación audiovisual } \\
\text { (Contenido obligatorio común) }\end{array}$ & $\begin{array}{l}\text { - Organización y gestión del sistema comunicativo atendiendo a sus parámetros } \\
\text { económicos y culturales. } \\
\text { - Políiticas del audiovisual a escala europea. } \\
\text { - Sistemas políitcos, medios de comunicación y opinión pública. } \\
\text { - La realidad socio-comunicativa en el contexto español. } \\
\text { - Mecanismos legislativos y deontológicos de la información y la comunicación } \\
\text { audiovisual. }\end{array}$ \\
\hline 6 & $\begin{array}{l}\text { Análisis de los discursos } \\
\text { audiovisuales y de sus efectos } \\
\text { sociales (Contenido obligatorio } \\
\text { común) }\end{array}$ & $\begin{array}{l}\text { - Metodologías de análisis de los mensajes audiovisuales. } \\
\text { - Funciones comunicativas: información, persuasión, entretenimiento... } \\
\text { - Estudios de recepción } \\
\text { - Estudio de los procesos psico-sociales, cognitivos, emocionales de la comunicación } \\
\text { social en contextos específicos. } \\
\text { - Publicidad, relaciones públicas y estrategias comunicativas. } \\
\text { Asignaturas: Métodos de investigación en comunicación (6ECTS) y Procesos y efectos } \\
\text { mediáticos (6ECTS) }\end{array}$ \\
\hline
\end{tabular}




\section{tarbiya $\mathbf{4 8}$}

18 Procesos de ideación y narrativa - Escritura de guion para informativos, reportajes, audiovisual (Contenido documentales y ficción audiovisual.

obligatorio común) - Formas y técnicas del espectáculo.

- Fundamentos creativos y persuasivos de la creatividad publicitaria.

- Teorías del relato audiovisual, la dramaturgia y la puesta en escena.

- Ideación de nuevos formatos.

Asignaturas: Laboratorio de composición audiovisual (6ECTS), Narrativa audiovisual (6ECTS), Guion audiovisual (6ECTS).

30 Técnicas y procesos creativos de - Dirección audiovisual: cine, televisión, vídeo.

producción, realización y - Aplicaciones creativas básicas sobre escenografía, iluminación, dirección de

postproducción (Contenido fotografía, dirección de actores.

obligatorio común)

- Procesos de creación, registro y difusión de la producción radiofónica, discográfica y otros productos sonoros.

- Diseño de productos multimedia y entornos informáticos interactivos.

- Edición y posproducción sonora, audiovisual y multimedia.

Asignaturas: Laboratorio de creación de audiovisuales de Ficción (6ECTS), Laboratorio de dirección y realización de TV (6ECTS), Laboratorio de dirección y producción de contenidos radiofónicos (6ECTS), Laboratorio de contenidos interactivos y transmedia (6ECTS), Laboratorio de fotografía (6ECTS).

12 Organización y producción - Diseño y evaluación de la gestión productiva en sus diversos formatos y soportes audiovisual y multimedia sonoros, visuales, audiovisuales, multimedia e interactivos

(Contenido obligatorio común) - Conocimiento de los sistemas de ayuda a la formación profesional, así como al desarrollo de guiones y proyectos, producción, distribución y exhibición de los diversos productos.

- Mercado audiovisual: estudios de creación e investigación de empresas.

- Estrategias de programación en radio y televisión.

- Investigación de audiencias.

- Gestión de campos virtuales y espacios físicos interactivos.

Asignaturas: Producción y gestión audiovisual (6ECTS), Investigación de audiencias (6ECTS).

\begin{tabular}{ll}
\hline Industrias culturales y & - Conocimiento del estado del mundo y su evolución reciente en los parámetros \\
movimientos estéticos & económicos, sociales y culturales básicos. \\
contemporáneos (Contenido & - Tendencias de las industrias culturales. Impacto de las tecnologías informativas y \\
obligatorio común) & comunicativas en la creación y gestión de nuevos ámbitos de la comunicación \\
& (museos, gabinetes, agencias, proyectos on-line). \\
& - Movimientos artísticos contemporáneos. \\
& - Globalización e interculturalidad. \\
& Asignaturas: Industrias culturales creativas (6ECTS), Tendencias del cine \\
& contemporáneo (6ECTS).
\end{tabular}




\section{taphiyg $\mathbf{4 8}$}

\begin{tabular}{ll}
\hline Tecnologías de los medios & - Conocimiento y aplicación de las tecnologías y de los sistemas utilizados para \\
audiovisuales y multimedia & procesar, elaborar y transmitir en los medios audiovisuales y multimedia. \\
(Contenido obligatorio común) & - Conocimiento del diseño y de los aspectos formales y estéticos en medios \\
& audiovisuales y entornos digitales. \\
& - Documentación en soportes analógicos y sistemas digitales. \\
& - Conocimiento de los aspectos tecnológicos y de gestión relacionados con todos los \\
& servicios integrados en la producción audiovisual y las telecomunicaciones. \\
& Asignaturas: Tecnologías de edición y postproducción audiovisual (6ECTS), \\
& Introducción a las tecnologías de la producción audiovisual (6ECTS), Tecnologías de \\
& la iluminación (6ECTS), Tecnologías de sonido (6ECTS). \\
\hline Escritura y expresión oral & - Aplicación práctica de la expresión escrita y oral a los medios de comunicación \\
(Contenido Obligatorio) ${ }^{5}$ & multimedia y soportes digitales. \\
& - Estrategias de comunicación oral. Locución y \\
& presentación en radio y televisión. \\
& - Inglés profesional en el ámbito de la comunicación. \\
& Asignatura: Laboratorio de escritura y expresión oral (6ECTS)
\end{tabular}

Fuente: Elaboración propia a partir del Libro Blanco de los estudios de Comunicación (2005) y de la Memoria del Grado en Comunicación y Creación Audiovisual (Plan 2016-2017).

Tabla 2. Distribución de créditos en las materias obligatorias del Grado en Comunicación y Creación Audiovisual propuesto en el Libro Blanco (2005) y las asignaturas obligatorias finalmente presentes en el Grado en Comunicación y Creación Audiovisual de la Universidad de Salamanca (CAAV).

\section{Universidades que ofertan Comunicación Audiovisual en España}

En prácticamente todas las Comunidades Autónomas de España se puede cursar el Grado de Comunicación Audiovisual, bien en un centro público o en uno privado. Por ejemplo, en la Comunidad de Madrid se puede estudiar en la Universidad Complutense de Madrid, una de las más veteranas y que mayor número de plazas oferta cada curso, con unas 270 por año académico; en la Universidad Carlos III de Madrid, que dispone de modalidad bilingüe; 0 en la Universidad Rey Juan Carlos, en la que se oferta el doble Grado en Periodismo y Comunicación Audiovisual y el Grado en Ingeniería de Sistemas Audiovisuales. En la comunidad andaluza se puede cursar el Grado en Comunicación Audiovisual en la Universidad de Sevilla (US), Universidad de Málaga (UMA) y Universidad de Granada (UGR), en la que han implantado nuevas modalidades relacionadas con su adscripción a la Facultad de Comunicación y Documentación. En Barcelona, la oferta es diversa, pero entre las públicas destacan la Universitat Pompeu Fabra, nombrada "la universidad española más productiva" por la Fundación BBVA, y la Universidad Ramón Llull. Esta es solo una pequeña muestra de toda la amplia oferta para cursar el Grado en Comunicación Audiovisual en España.

Los criterios de calidad académicos pueden ser uno de los factores determinantes a la hora de decidir en qué universidad matricularse. Estos criterios pueden ser consultados por los ciudadanos en las diferentes universidades, pues son un dato de dominio público y que da transparencia a la propia universidad ${ }^{6}$. Otra forma de acceder a esta

\footnotetext{
${ }^{5}$ En la memoria del Grado en Comunicación y Creación Audiovisual aparece como "Escritura y expresión oral. Adecuación de su uso a los medios de comunicación sonoros y audiovisuales".

${ }^{6}$ Por ejemplo, la Unidad de Evaluación de la Calidad de la Universidad de Salamanca se hace eco de los diferentes ránquines mundiales de universidades. Así, la Universidad de Salamanca se mantiene entre las
} 
información de manera más sencilla pueden ser los ránquines de los diferentes medios de comunicación, como el que anualmente publica el periódico generalista EI Mundo. En el listado que publicaron en el año 2019, las cinco primeras universidades fueron: Universidad Autónoma de Barcelona; Universidad Carlos III de Madrid; Universidad de Navarra; Universidad Pompeu Fabra; y, por último, Universidad Rey Juan Carlos (El Mundo, 2019). Otro factor que influya en la elección de la universidad puede ser el precio del curso. De estas cinco, la más cara es la Universidad de Navarra, que es privada, y las más económicas por año académico son las dos universidades de la Comunidad de Madrid.

\section{Grado de Comunicación y Creación Audiovisual en Castilla y León}

La transferencia de las competencias en materia universitaria del Estado (Ministerio de Educación) a la Comunidad Autónoma de Castilla y León en el año 1995 tuvo como consecuencia la creación de un marco legal y normativo regulador del sistema universitario en la Comunidad al amparo de la legislación nacional y comunitaria. A partir de entonces, la Junta de Castilla y León asumió funciones gestoras y creó un distrito universitario único que albergaba las nueve provincias de la Comunidad. Los potenciales alumnos y alumnas, independientemente de su lugar de residencia pueden elegir ser candidatos/as y finalmente alumnos/as en cualquiera de las cuatro universidades públicas, Universidad de Salamanca, Universidad de Valladolid, Universidad de León y Universidad de Burgos, y en las Universidades privadas.

Las principales normativas de la comunidad van ${ }^{7}$ desde el Decreto 233/1995, de 16 de noviembre, por el que se crean o transforman centros y se autorizan enseñanzas en las Universidades en León, Salamanca y Valladolid hasta la Orden EDU/1006/2014, de 21 de noviembre, por la que se regula el reconocimiento de Unidad de Investigación Consolidada de Castilla y León; pasando por los Decretos 141/1996, de 23 de mayo, por los que se crean, transforman y adscriben centros y se autorizan estudios en las Universidades de Burgos, León y Valladolid; el Decreto 226/1996 de 26 de septiembre por el que se autorizan estudios y se crean, transforman 0 cambian de denominación Centros en las Universidades de Valladolid, León y Burgos; el Decreto 19/1997, de 6 de febrero y el Decreto180/1997, de 26 de septiembre, por el que se autorizan estudios y se crean, transforman y cambian de denominación Centros en las Universidades de Salamanca, Valladolid, León y Burgos; 0 el Decreto 65/2013, de 3 de octubre, por el que se regula la creación, modificación y supresión de las Escuelas de Doctorado en Universidades de Castilla y León.

Con la Ley 12/2010, de 28 de octubre, por la que se modifica la Ley 3/2003, de 28 de marzo, de Universidades de Castilla y León, se legisla el Consejo de Universidades de Castilla y León, como "órgano colegiado de consulta y

500 mejores universidades del mundo en diferentes disciplinas según el prestigioso Shanghai Ranking's Global Ranking of Academic Subjects 2019, entre ellas la que aquí nos ocupa, Comunicación. Destaca, sobre todo, en la materia Food Science Technology, de la rama de conocimiento Engineering, donde ocupa el intervalo 51-75 y figura, por tanto, entre las 100 mejores universidades del mundo en este campo. La siguiente área mejor situada es precisamente Comunicación -Communication-, situándose entre las 300 mejores instituciones del mundo (intervalo 201-300). Dentro de la rama de conocimiento Natural Sciences, la Universidad de Salamanca se sitúa en el intervalo 301-400 en la materia Earth Sciences, y 401-500 en las materias Physics y Biotechnology. Adicionalmente, en la rama de conocimiento Life Sciences, la USAL destaca en la materia Biological Sciences, en el intervalo 401-500. Por último, las materias Education y Business Administration se sitúan en el intervalo 301-400. Además, en la docencia ocupa el puesto 76-100 en el Ranking de Enseñanza Europea, Times Higher Education (THE) Europe Teaching Rankings 2019, situándose así entre las 100 mejores universidades para la docencia de excelencia en Europa. Por otro lado, se sitúa entre las 500 mejores instituciones del mundo según la vigésima edición del Ranking Web de Universidades de 2019. En concreto, alcanza la posición número 486, que la ubica en el puesto 14 en España y 189 en Europa.

${ }^{7}$ Para referencia completa de las normativas ver Tabla 3. 


\section{taphiya $\mathbf{4 8}$}

asesoramiento para la programación, ordenación y planificación universitaria, en orden a procurar la máxima coordinación académica entre las Universidades" (BOE, núm. 97, de 23 de abril, p. 15784), organizado en un pleno y dos comisiones (Comisión Académica y Comisión de Consejos Sociales); la programación universitaria de Castilla y León; la creación y reconocimiento de Universidades; creación, reconocimiento, modificación y supresión de centros universitarios y enseñanzas; adscripción de centros de enseñanza universitaria a universidades públicas; creación, supresión y adscripción de Institutos Universitarios de Investigación; y registro de universidades, centros y enseñanzas, entre otros.

Al Consejo de Universidades de Castilla y León se le atribuyen, entre otras, y tal y como se recoge en el Artículo 7 (Ley 3/2003, de 28 de marzo, de Universidades de Castilla y León), las siguientes funciones: conocer la Programación Universitaria de Castilla y León, apoyar mecanismos de coordinación interuniversitaria que favorezcan la participación de la sociedad en las Universidades para la ejecución de programas de interés general, promover actividades que potencien las relaciones de las Universidades con la sociedad, informar el Mapa de Titulaciones Oficiales de Castilla y León y la programación de oferta de enseñanzas de las Universidades, así como la planificación de estudios de interés para la comunidad, conocer las directrices básicas a seguir por la Junta de Castilla y León y las Universidades en la ordenación de becas, créditos y ayudas y en la regulación de precios públicos por la prestación de servicios académicos, conocer las actividades de extensión universitaria desarrolladas por las Universidades y las programadas por la Junta de Castilla y León, buscando la coordinación de todas ellas.

A nivel autonómico, en Castilla y León la enseñanza universitaria se regula por la siguiente normativa que recogemos en la Tabla 3.

\begin{tabular}{|c|c|c|}
\hline \multirow{2}{*}{$\begin{array}{l}\text { Legislación autonómica } \\
\text { básica sobre } \\
\text { universidades (Castilla y } \\
\text { León) }\end{array}$} & LEY 3/2003, de 28 de marzo, de Universidades de Castilla y León & $\begin{array}{l}\text { BOCYL: } 4 / 04 / 2003 \\
\text { BOE: } 23 / 04 / 2003\end{array}$ \\
\hline & $\begin{array}{l}\text { Ley 12/2010, de } 28 \text { de octubre, por la que se modifica la Ley 3/2003, de } \\
28 \text { de marzo, de Universidades de Castilla y León }\end{array}$ & $\begin{array}{l}\text { BOCYL: } 10 / 11 / 2010 \\
\text { BOE: } 23 / 11 / 2010\end{array}$ \\
\hline \multirow[t]{4}{*}{ Normas generales } & $\begin{array}{l}\text { RESOLUCIÓN de } 8 \text { de abril de 2014, del Rectorado de la Universidad de } \\
\text { Valladolid, por la que se acuerda la publicación del procedimiento para la } \\
\text { solicitud de adaptaciones en las pruebas de acceso a las enseñanzas } \\
\text { universitarias oficiales de grado en las Universidades Públicas de Castilla y } \\
\text { León para estudiantes de Bachillerato o Ciclos Formativos de Grado } \\
\text { Superior que presentan necesidades educativas especiales u otras } \\
\text { debidamente justificadas }\end{array}$ & BOCyL:22/04/2014 \\
\hline & $\begin{array}{l}\text { ORDEN EDU/213/2014, de } 27 \text { de marzo, por la que se desarrolla el } \\
\text { Decreto 64/2013, de } 3 \text { de octubre, de ordenación de las enseñanzas } \\
\text { universitarias oficiales de Grado y Máster en el ámbito de la Comunidad } \\
\text { de Castilla y León }\end{array}$ & BOCyL:4/04/2014 \\
\hline & $\begin{array}{l}\text { DECRET0 67/2013, de } 17 \text { de octubre, por el que se desarrolla la } \\
\text { regulación del régimen del personal docente e investigador contratado en } \\
\text { las Universidades Públicas de Castilla y León }\end{array}$ & BOCyL:21/10/2013 \\
\hline & $\begin{array}{l}\text { DECRETO 64/2013, de } 3 \text { de octubre, de ordenación de las enseñanzas } \\
\text { universitarias oficiales de grado y máster en el ámbito de la Comunidad } \\
\text { de Castilla y León }\end{array}$ & BOCyL:7/10/2013 \\
\hline
\end{tabular}




\section{tarbiya $\mathbf{4 8}$}

ORDEN EDU/411/2012, de 8 de junio, por la que se regula el

BOCyL:18/06/2012

procedimiento por el que las Universidades de Castilla y León pueden

obtener autorización para la impartición de la formación equivalente a la

formación pedagógica y didáctica exigida para aquellas personas que, estando en posesión de una titulación declarada equivalente a efectos de docencia, no pueden realizar los estudios de máster

ORDEN EDU/419/2010, 29/3, por la que se determinan los porcentajes BOCyL: 9/04/2010 de plazas a reservar a determinados grupos de estudiantes en el procedimiento de admisión a la Universidad

ORDEN EDU/2017/2009, de 15 de octubre, por la que se crea la comisión BOCyL:28/10/2009 organizadora de las pruebas de acceso a las enseñanzas universitarias oficiales de grado, y se regulan ciertos aspectos para el desarrollo de dichas pruebas. Corrección de errores publicada en BOCYL 11/08/2008. Modificada por Orden EDU/273/2011. BOE 24/03/2011

DECRETO 104/1997, de 8 de mayo, por el que se implanta el distrito BOCyL: 8/05/1997 único universitario de Castilla y León y se crea su Comisión coordinadora

DECRETO 97/1987, de 24 de abril, por el que se establecen criterios BOCyL: 24/04/1987 reguladores de las convocatorias de ayuda a la investigación, proyectos y programas de investigación científica y técnica, formación de investigadores y becas
Centrosy enseñanzas universitarios
ORDEN EDU/1006/2014, de 21 de noviembre, por la que se regula el reconocimiento de Unidad de Investigación Consolidada de Castilla y León
BOCyL:7/10/2013

65/2013, de 3 de octubre, por el que se regula la creación, modificación y supresión de Escuelas de Doctorado en Universidades de Castilla y León

ORDEN EDU/995/2013, de 26 de noviembre, por la que se desarrolla el Decreto 65/2013, de 3 de octubre, por el que se regula la creación, modificación y supresión de Escuelas de Doctorado en Universidades de Castilla y León

ORDEN EDU/641/2012, de 25 de julio, por la que se regula la realización de las prácticas de las asignaturas del practicum de las enseñanzas universitarias de Grado en Educación Infantil y Grado en Educación Primaria en centros docentes sostenidos con fondos públicos de la Comunidad de Castilla y León que imparten estas enseñanzas y se delega la competencia para dictar resolución sobre el reconocimiento de los centros de formación en prácticas y sobre la acreditación de los maestros tutores de prácticas BOCyL: 31/07/2012 A. 109/2009, 24/09, por el que se autoriza la implantación de Enseñanzas BOCyL: 30/09/2009 Universitarias oficiales en la Universidad de Valladolid

DECRETO 180/1997, de 26 de septiembre, por el que se autorizan BOCYL: 26/09/1997 estudios y se crean, transforman y cambian de denominación Centros en las Universidades de Salamanca, Valladolid, León y Burgos 


\section{tapbiya $\mathbf{4 8}$}

DECRETO 19/1997, de 6 de febrero, por el que se autorizan estudios y se $\quad$ BOCyL: 6/02/1997
transforman Centros en las Universidades de Salamanca, León y Burgos

DECRETO 226/1996, de 26 de septiembre, por el que se autorizan BOCyL: 26/09/1996

estudios y se crean, transforman o cambian de denominación Centros en

las Universidades de Valladolid, León y Burgos

DECRETO 174/1996, de 4 de julio, por el que se autoriza a la Universidad BOCyL: 4/07/1996

de Valladolid a integrar a la Escuela Universitaria de Magisterio de

Segovia "Nuestra Señora de la Fuencisla"

DECRETO 141/1996, de 23 de mayo, por el que se crean, transforman y BOCyL: 3/05/1996

adscriben Centros y se autorizan estudios en las Universidades de Burgos,

León y Valladolid

DECRETO 233/1995, de 16 de noviembre, por el que se crean o BOCyL: 16/11/1995

transforman centros y se autorizan enseñanzas en las Universidades de

León, Salamanca y Valladolid

Tabla 3. Legislación aplicable en la CCAA de Castilla y León en materia de Universidad y estudios universitarios.

Tres de las cuatro Universidades públicas de la Comunidad Autónoma de Castilla y León cuentan con estudios de "Comunicación". Entre las más veteranas están la Universidad de Burgos y la Universidad de Valladolid, aunque ésta oferta el Grado en Periodismo y no en Comunicación Audiovisual. Además, es significativo que, mientras la gran mayoría de los Grados en Comunicación suelen depender de Facultades de Ciencias Sociales o Ciencias de la Información, en esta Universidad depende de la Facultad de Filosofía y Letras. Sin embargo, habría que indicar que la Universidad de Salamanca comenzó a impartir los estudios de Comunicación Audiovisual como Licenciatura de segundo ciclo en el curso 1994-1995, convirtiéndose en el programa de comunicación más antiguo de las universidades públicas de Castilla y León y uno de los más antiguos de España. Desde entonces, la Licenciatura se ha impartido con tres planes de estudio: el fundacional (aprobado mediante Resolución de 4 de octubre de 1994, BOE 21/10/1994), el que renovó su estructura curricular seis años después (aprobado por Resolución de 28 de julio de 2000, BOE 24/08/2000, y modificado después en algunos aspectos por la Orden ECD/3455/2002 y la Resolución de 10 de marzo de 2003, BOE 18/01/2003 y 27/03/2003, respectivamente) y el actual Grado en Comunicación y Creación Audiovisual (Plan 20162017), publicado en el BOCYL Número 231, de viernes 1 de diciembre de 2018. El desarrollo del Grado en Comunicación Audiovisual tuvo lugar entre 2010 y 2017, cuando se matriculó la última promoción que cursó el plan de estudios de 2010. Tal y como sucedió en el curso 2010-2011, en el 2017-2018, se produjo la entrada en vigor del nuevo Grado en Comunicación y Creación Audiovisual, que vino a incorporar los requerimientos de la Agencia para la Calidad del Sistema Universitario de Castilla y León (ACSUCYL) tras el proceso negativo de evaluación del título anterior. La

\footnotetext{
${ }^{8}$ Para poder cursar el segundo ciclo de la Licenciatura en Comunicación Audiovisual había que tener cursado el primer ciclo de una Licenciatura afín. Así, como se indica en la Resolución de 4 de octubre de 1994, BOE 21/10/1994: "podrán acceder al segundo ciclo de la Licenciatura en Comunicación Audiovisual. además de quienes hayan cursado el primer ciclo de estos estudios: a) Quienes hayan superado el primer ciclo de la Licenciatura en Periodismo o de la Licenciatura en Publicidad y Relaciones Públicas. b) Quienes están en posesión de un título universitario de carácter oficial o hayan superado un primer ciclo de estudios universitarios oficiales, cursando como complementos de formación, de no haberlo hecho antes, 12 créditos en Comunicación e Información Audiovisual; 10 créditos en Teoría de la Comunicación y Teoría de la Información, y 8 créditos en Lengua".
} 


\section{taphiya $\mathbf{4 8}$}

implantación completa de este nuevo grado ha sido progresiva, con un curso cada año académico (en 2017-2018 se implantó primero, en 2018-2019 se implantó segundo y así sucesivamente) y, de igual manera, dejarán de impartirse los cursos del anterior grado.

Por su parte, tres de las cinco Universidades privadas de Castilla y León ofrecen titulaciones oficiales de Grado en el área de "Comunicación / Periodismo / Publicidad y Relaciones Públicas". La Universidad Pontificia de Salamanca implantó el Grado en Comunicación Audiovisual en 2009, además del de Periodismo y el de Publicidad y Relaciones Públicas, al igual que Universidad Europea Miguel de Cervantes.

\begin{tabular}{llll}
\hline Universidad & Sede & Facultad & Título y año de implantación \\
\hline Burgos & Burgos & $\begin{array}{l}\text { Facultad de Ciencias Sociales y } \\
\text { Jurídicas }\end{array}$ & Grado en Comunicación Audiovisual (2009) \\
\hline Salamanca & Salamanca & Facultad de Ciencias Sociales & Grado en Comunicación Audiovisual (2010) \\
& Grado en Comunicación y Creación Audiovisual (2017) \\
\hline Valladolid & Facultad de Ciencias Sociales, & Grado en Publicidad y RelacionesPúblicas (2010) \\
& Jurídicas y de la Comunicación & Doble Grado Publicidad y Relaciones Públicas y \\
& & Turismo \\
\cline { 2 - 4 } & Valladolid & Facultad de Filosofía y Letras & Grado en Periodismo (2009) \\
\hline $\begin{array}{l}\text { Fuente: elaboración propia a través de página web de ANECA y ACSUCYL } \\
\text { https://srv.aneca.es/ListadoTitulos/ } \\
\text { http://seguimiento2.acsucyl.com/AcsucylWeb/buscadorTitulos/busqueda.jsp }\end{array}$ & \\
\hline
\end{tabular}

Tabla 4. Universidades públicas de Castilla y León que cuentan con Títulos de Grado en Comunicación / Periodismo / Publicidad y Relaciones Públicas.

\begin{tabular}{llll}
\hline Universidad & Sede & Facultad & Título y año de implantación \\
\hline IE University & Segovia & $\begin{array}{l}\text { Facultad de Ciencias Sociales y } \\
\text { Jurídicas }\end{array}$ & $\begin{array}{l}\text { Grado en Comunicación y Medios Digitales - Bachelor } \\
\text { in Communication and Digital Media (2017) }\end{array}$ \\
\hline $\begin{array}{lll}\text { Universidad Pontificia } \\
\text { de Salamanca }\end{array}$ & Salamanca & Facultad de Comunicación & Grado en Comunicación Audiovisual (2009) \\
& & Grado en Periodismo (2009) \\
& & Grado en Publicidad y Relaciones Públicas (2009) \\
& Grado en Marketing y Comunicación (2014) \\
& Doble Grado en Comunicación Audiovisual y \\
& Periodismo \\
& Doble Grado en Publicidad y RR.PP. y Marketing y \\
& Comunicación
\end{tabular}




\section{taphiya $\mathbf{4 8}$}

\begin{tabular}{|c|c|c|c|}
\hline $\begin{array}{l}\text { Universidad Europea } \\
\text { Miguel de Cervantes }\end{array}$ & Valladolid & $\begin{array}{l}\text { Facultad de Ciencias Sociales y } \\
\text { Jurídicas }\end{array}$ & $\begin{array}{l}\text { Grado en Periodismo (2009) } \\
\text { Grado en Comunicación Audiovisual (2009) } \\
\text { Grado en Publicidad y Relaciones Públicas (2009) } \\
\text { Doble Grado en Administración y Dirección de } \\
\text { Empresas y Publicidad y Relaciones Públicas } \\
\text { Doble Grado en Periodismo y Comunicación } \\
\text { Audiovisual }\end{array}$ \\
\hline
\end{tabular}

Fuente: elaboración propia a través de página web de ANECA y ACSUCYL

https://srv.aneca.es/ListadoTitulos/

http://seguimiento2.acsucyl.com/AcsucylWeb/buscadorTitulos/busqueda.jsp

Tabla 5. Universidades privadas de Castilla y León que cuentan con Títulos de Grado en Comunicación / Periodismo / Publicidad y Relaciones Públicas.

La oferta tanto pública como privada es amplia como consecuencia de que el área sea un campo en expansión en la sociedad de la información y, por consiguiente, la demanda por parte de los alumnos y las alumnas es elevada. A modo de ejemplo, se muestran los datos de plazas ofertadas, estudiantes matriculados, nota de corte y nota media de acceso de la Universidad de Salamanca. A tenor de estos datos, se puede observar cómo el acceso a las titulaciones de Comunicación Audiovisual en esta universidad ha tenido históricamente una buena aceptación en cuanto a número de matrículas y notas de corte, pues siempre ha cubierto las plazas ofertadas (en la mayoría de los cursos, en el primer plazo de matrícula) y la nota media de acceso siempre ha estado por encima de la de corte. Por tanto, aquellos alumnos que no realizan el examen de la Evaluación de Bachillerato para el Acceso a la Universidad (EvAU) en la primera convocatoria tienen difícil el acceso al Grado de Comunicación Audiovisual y de Comunicación y Creación Audiovisual, por lo que puede decirse que solo accede al Grado el alumnado con calificaciones más notables.

\begin{tabular}{|c|c|c|c|c|c|c|}
\hline Año & Centro & Titulación & $\begin{array}{l}\text { Plazas } \\
\text { ofertadas }\end{array}$ & $\begin{array}{l}\text { Estudiantes que } \\
\text { acceden al título } \\
\text { (incluye } \\
\text { adaptaciones a } \\
\text { Grado y traslados) }\end{array}$ & $\begin{array}{l}\text { Nota de } \\
\text { corte }\end{array}$ & $\begin{array}{l}\text { Nota media } \\
\text { de acceso }\end{array}$ \\
\hline $2010-11$ & $\begin{array}{l}\text { Facultad de } \\
\text { Ciencias } \\
\text { Sociales } \\
\text { (FCCSS) }\end{array}$ & $\begin{array}{l}\text { Grado en Comunicación } \\
\text { Audiovisual (CAV) }\end{array}$ & 60 & 62 & 8.40 & $9,23^{9}$ \\
\hline 2011-12 & FCCSS & CAV & 60 & 69 & 8,20 & 9,27 \\
\hline $2012-13$ & FCCSS & CAV & 60 & 53 & 7,26 & 9,50 \\
\hline 2013-14 & FCCSS & CAV & 60 & 64 & 8,464 & 9,13 \\
\hline 2014-15 & FCCSS & CAV & 60 & 64 & 8,358 & 9,26 \\
\hline 2015-16 & FCCSS & CAV & 60 & 62 & 7,67 & 9,35 \\
\hline
\end{tabular}

\footnotetext{
${ }^{9}$ Con la reforma de la selectividad la nota de corte pasa a ser sobre 10 a ser sobre 14 , lo que sucede a partir del curso 2010-2011.
} 


\section{taphiya $\mathbf{4 8}$}

\begin{tabular}{lllllll}
\hline 2016-17 & FCCSS & CAV & 60 & 61 & 8,330 & 9,40 \\
\hline $2017-18$ & FCCSS & $\begin{array}{l}\text { Graduado o Graduada en } \\
\text { Comunicación y Creación } \\
\text { Audiovisual (CCAV) }\end{array}$ & 60 & 67 & 7,896 & 9,17 \\
\hline $2018-19$ & FCCSS & CCAV & 60 & 71 & 8,756 & 9,84 \\
\hline $2019-20$ & FCCSS & CCAV & 60 & 68 & 5,46 & 9,82 \\
\hline
\end{tabular}

Fuente: Elaboración propia a partir de los datos del portal de transparencia

Tabla 6. Notas de acceso y corte de los estudios de comunicación en la Universidad de Salamanca.

\section{Competencias y salidas profesionales: análisis del Grado en Comunicación y Creación Audiovisual de la Universidad de Salamanca}

El Grado en Comunicación y Creación Audiovisual tiene un enfoque generalista que proporciona a sus graduados la posibilidad de dedicarse profesionalmente a cualquier ámbito relacionado con la comunicación audiovisual. Estos estudios capacitan al estudiante para realizar labores de investigador, docente y experto en estudios de comunicación, para entender y analizar las políticas culturales y creativas, conocer la prospectiva sectorial y económica de los medios de comunicación, así como para desempeñar tareas de otros perfiles laborales como las del realizador y/o productor audiovisual, entre otros.

El Grado en Comunicación y Creación Audiovisual no solo capacita a los estudiantes para utilizar los diversos instrumentos técnicos-tecnológicos, sino también para crear historias originales e interesantes (de ficción, informativas, documentales, etc.) audiovisuales, radiofónicas, sonoras, escritas, digitales, etc., destinadas a cine, radio, televisión, medios sociales, blogs o webs de descargas. Además de ello, y según la estructura de los nuevos grados adaptados al Espacio Europeo de Educación Superior (EEES), existe la posibilidad de obtener una especialización a través de las asignaturas optativas elegidas de entre las ofertadas en los distintos cursos académicos, lo que se reflejará en el expediente académico.

Sirvan de ejemplo los objetivos fundamentales propuestos para el Grado en Creación y Comunicación Audiovisual de la Universidad de Salamanca recogidos en la Memoria de Verificación aprobada por la Agencia para la Calidad del Sistema Universitario de Castilla y León (ACSUCYL) el 10 de julio de 2017:

- El Grado en Comunicación y Creación Audiovisual ha de fomentar la capacidad crítica, analítica y reflexiva en relación con el hecho audiovisual, con un conocimiento técnico y estético de las formas, procesos y tendencias de la comunicación visual de nuestro entorno.

- El estudiante de Comunicación y Creación Audiovisual ha de adquirir un conocimiento básico y generalista de los principales acontecimientos y mensajes que configuran el panorama actual de los medios audiovisuales, de las representaciones icónicas y acústicas y de su evolución en las sociedades contemporáneas.

- El Grado en Comunicación y Creación Audiovisual debe proporcionar un conocimiento exhaustivo de las técnicas y procesos de creación y difusión audiovisuales en sus diversas fases, así como de las interrelaciones entre los sujetos de la comunicación audiovisual: autores, instituciones, empresas, medios, soportes y receptores. Esta formación capacitará al graduado para la toma de decisiones creativas y profesionales en el campo de la comunicación audiovisual y de la gestión de los recursos tecnológicos y humanos en las empresas del sector.

- L Los graduados en Comunicación y Creación Audiovisual deben conocer las metodologías y conceptos aplicables 


\section{taphiya $\mathbf{4 8}$}

en las diferentes ramas de investigación, desarrollo e innovación de la comunicación audiovisual y los futuros entornos tecnológicos.

- L Los graduados en Comunicación y Creación Audiovisual deberán ser capaces de expresarse con claridad y coherencia en las lenguas propias de su comunidad, así como tener conocimiento de otros idiomas, en particular el inglés, para comunicarse en su ejercicio profesional.

Estos objetivos podrán alcanzarse con la adquisición de los siguientes saberes y habilidades:

- Formación en Ciencias Sociales, Humanidades y Ciencia y Tecnología para proporcionar una competencia contextual básica de naturaleza interdisciplinar.

- Formación en teoría, historia y estructura de la comunicación y conocimiento en los fundamentos teóricos y prácticos, y los métodos más habituales en los procesos de creación, producción, distribución, recepción y evaluación de los mensajes y discursos comunicativos. Los parámetros económicos, sociales, culturales y políticos son fundamentales para esta formación, con el objeto de proporcionar un conocimiento integral de la estructura del sistema audiovisual.

- Formación en las capacidades expresivas y en los lenguajes de cada uno de los medios y formatos de la comunicación -fotografía, cine, televisión, radio, vídeo, entornos en red y soportes multimedia-, así como una capacidad de análisis-linguístico, pragmático, estético e ideológico- de la producción audiovisual tanto en medios icónicos como en los entornos digitales.

- Formación en el conocimiento y uso de las tecnologías de la comunicación en los distintos entornos multimedia e hipermedia para su aplicación en el ámbito de la comunicación audiovisual y el desarrollo de nuevos formatos y soportes audiovisuales y en red.

- Formación ética profesional en el quehacer comunicativo a través del conocimiento de los códigos deontológicos y los mecanismos legislativos que afectan a los diversos ámbitos de la comunicación, fomentando además el desarrollo de una conciencia de responsabilidad social y cultural.

- Formación básica relacionada con la planificación y gestión de empresas audiovisuales públicas y privadas y la realización de proyectos comunicativos innovadores y adaptados al entorno.

- Formación básica en la metodología y las técnicas de investigación y de análisis aplicadas en los diversos ámbitos de la comunicación audiovisual y tecnológica. Iniciación a la metodología y las técnicas aplicadas en la investigación de las audiencias y los hábitos de uso y consumo de las mismas.

Por tanto, consideramos que la preparación que adquieran los estudiantes que cursen el Grado en Comunicación Audiovisual o cualquiera de los Grados afines como Grado en Comunicación y Creación Audiovisual, Grado en Periodismo, Grado en Publicidad, etc. los habilitará para desarrollar las competencias adquiridas y aplicarlas en el mundo profesional, un entorno laboral cambiante y exigente que requiere habilidad para desarrollar diferentes tareas y ser trabajadores multidisciplinares. Deberán saber planificar de manera eficiente recursos humanos y técnicos en aras de una mayor rentabilidad. Además, tendrán que desarrollar su trabajo en diferentes medios y soportes como prensa escrita, radio, televisión, cine, etc. sin obviar las nuevas tecnologías, lo que les exige un aprendizaje constante. No solo podrán ser creadores, sino también analizar relatos audiovisuales, pues una de las competencias que más se debe trabajar en grados como Comunicación Audiovisual es la formación de ciudadanos críticos. Para ello, es fundamental trabajar y desarrollar competencias de alfabetización mediática, pues tan importante es saber crear como saber analizar, algo que debería trabajarse desde los niveles más bajos de la educación y afianzarse con mensajes más complejos a medida que aumenta el nivel de estudios de los alumnos.

Son varias las salidas profesionales a las que los estudiantes de Comunicación Audiovisual pueden optar. Por un lado, 
está la enseñanza, que puede ser dirigida hacia el ámbito universitario: bien realizando una Tesis Doctoral, tras cursar un Máster, u optando a alguna de las plazas de Profesor Asociado con la que las universidades contratan a profesores especialistas en una determinada materia. Otra opción es realizar el Máster de Profesor de Educación Secundaria Obligatoria y Bachillerato, Formación Profesional y Enseñanzas de Idiomas que habilita al estudiante para impartir clases en Educación Secundaria y en Formación Profesional, en los ciclos de Grado Medio y Grado Superior de la familia de Imagen y Sonido ${ }^{10}$.

Las salidas profesionales más habituales suelen estar en los medios de comunicación, como en la televisión, que generalmente es la más demandada, seguida de la radio y, en menor medida, en la prensa escrita, en la que suelen trabajar los Graduados en Periodismo. Los Graduados en Comunicación Audiovisual también pueden encontrar trabajo en empresas de comunicación, publicidad y márquetin. Gracias a la enorme diversidad de optativas que se ofertan, los alumnos pueden ampliar su abanico laboral en un sector en auge como el desarrollo de productos de animación, videojuegos o aplicaciones para móviles, siendo una opción muy demanda en la actualidad. Aunque no suele ser una de las salidas más habituales, los estudiantes también pueden acceder a puestos en editoriales, ya que están capacitados para crear novelas gráficas, comunicación corporativa, etc., u organizar eventos, entre otras labores. Un medio muy deseado por los estudiantes suele ser el cine, a pesar de que su inserción laboral es más complicada ya que se trata de un campo con un sistema de incorporación más complejo. Sin duda alguna, la especialización es un plus que permite diferenciar a los y las estudiantes teniendo en cuenta la multidisciplinariedad que aporta una carrera como el Grado en Comunicación Audiovisual.

Por último, cabe destacar que los datos más recientes del Ministerio de Educación, Cultura y Deporte indican que, en el año 2014, estaban afiliados a la Seguridad Social el 55\% de los egresados de Comunicación Audiovisual en cualquier universidad española en el curso 2009-2010.

\section{Conclusiones}

En estas páginas se ha analizado qué se estudia en el Grado de Comunicación Audiovisual en España. Para analizarlo con mayor profundidad, se ha utilizado el ejemplo del Grado que se imparte en la Universidad de Salamanca, el más longevo de la Comunidad de Castilla y León y que goza de reconocimiento entre el alumnado, tal y como se ha visto con el número de alumnos que año tras año se matriculan. Cuando un alumno acaba sus estudios de Bachillerato se le presenta una oferta muy amplia y ecléctica de estudios, ya opten por una de las muchas carreras universitarias que se imparten en España o por un Grado Medio o Superior de Educación Profesional. Conocer qué se estudia, desde qué enfoque, si hay más asignaturas de corte profesional o teórico, el grado de empleabilidad, las competencias a desarrollar, etc. resulta fundamental para realizar una buena elección. Este artículo pretende facilitar el trabajo a aquellos que estén pensando en elegir estudios de Comunicación Audiovisual, así como ofrecer un panorama general y el análisis de un caso concreto a todo aquel vinculado a esta área.

\footnotetext{
${ }^{10}$ Se puede ver más información sobre la familia de Imagen y Sonido en el siguiente enlace: https://www.todofp.es/que-como-y-donde-estudiar/que-estudiar/familia/loe/imagen-sonido.html
} 


\section{Bibliografía}

agencia nacional de Evaluación de la Calidad y la Acreditación (AneCA) (2005). Libro Blanco. Títulos de Grado en Comunicación.

http://www.aneca.es/var/media/150336/libroblanco_comunicacion_def.pdf

DELORS, J. (1996). La educación encierra un tesoro. Informe de la UNESCO de la Comisión Internacional sobre la educación para elsiglo XXI, presidida por Jacques Delors. Santillana / Ediciones UNESCO.

EL Mundo (2019). Mejores Universidades. El Mundo

https://www.elmundo.es/especiales/ranking-universidades/donde-estudiar-comunicacion-audiovisual.html

RodríGuez MeRCHÁn, Eduardo (2007). La enseñanza del cine en España: perspectiva histórica y panorama actual. Comunicar: Revista científica iberoamericana de comunicación y educación, 29, 13-20. 


\section{taphiya $\mathbf{4 8}$}

Resumen.

El título de Licenciado en Comunicación Audiovisual fue regulado en 1991. Desde entonces, se han sucedido reformas en los planes de estudio y la organización universitaria encaminadas a adaptarse al Espacio Europeo de Educación Superior (EEES). Actualmente, el Libro Blanco de los títulos de Grado en Ciencias de la Comunicación (2005) enmarca las diferentes titulaciones que se pueden cursar en España. Se realiza aquí un repaso por planes de estudio, universidades, notas de acceso, legislación, competencias y salidas profesionales de las titulaciones del área de la Comunicación Audiovisual centrado en la Comunidad Autónoma de Castilla y León.

Palabras clave. Comunicación Audiovisual; España; Ciencias de la Comunicación; Universidad; Castilla y León.

\section{Abstract.}

The Degree in Audiovisual Communication was firstly regulated in 1991. From that moment on, reforms have been made in study plans and university organization in order to adapt the title to the European Higher Education Area (EHEA). Currently the White Book of bachelor's degrees in Communication Sciences (2005) frames the different degrees that can be coursed in Spain. This paper aims to be a review of study plans, universities, access notes, legislation, skills and professional opportunities of the degrees in the area of Audiovisual Communication with special emphasis on the Autonomous Community of Castilla y León.

Key-words. Audiovisual Communication; Spain; Communication Sciences; University; Castilla y León.

Beatriz González de Garay Domínguez Universidad de Salamanca bgonzalezgaray@usal.es

María Marcos Ramos Universidad de Salamanca

mariamarcos@usal.es 